\title{
TOWARDS ENERGY EFFICIENT CLOUD COMPUTING USING DEPENDENCY GRAPH
}

\author{
Preetilata Sahu \\ Vishwavidyalaya Engineering College, \\ Lakhanpur (C.G.) \\ Dept. of Computer Science and Engineering \\ Ambikapur, Chhattisgarh, India \\ Dr. Dipti Verma \\ Assistant Professor \\ Vishwavidyalaya Engineering College, \\ Lakhanpur (C.G.) \\ Dept. of Computer Science and Engineering \\ Ambikapur, Chhattisgarh, India
}

\begin{abstract}
-
Nowadays, research are being directed in the field of large system models. These large system models generally manages the distributed environment to break down and understand behavior of the systems. A large number of the system give simulation of the distributed environment to run their experiments. Hence, when there is an expansion in demand of cloud and distributed system, side by side it is important to maintain the power consumption and efficiency of the framework. This is a most challenging assignment to perform. In this paper, we give energy optimizing model with the help of migration manager to manage the productivity of the entire system. We will contrast DVFS algorithm with on Demand and Conservative mode of operation in cloud. We will exhibit our experiment in CloudSim software.
\end{abstract}

Keywords - Energy Saving, Power Consumption, DVFS, DAX, onDeman Mode, Conservative Mode, Migration Manager, CloudSim.

Cite this Article: Preetilata Sahu and Dipti Verma, Towards Energy Efficient Cloud Computing Using Dependency Graph, International Journal of Computer Engineering and Technology, 10(2), 2019, pp. 74-82.

http://iaeme.com/Home/issue/IJCET?Volume=10\&Issue=2 


\section{INTRODUCTION}

Cloud computing is a model for conveyance of services and access in which virtual and dynamically scalable resources are delivered as a services on demand over the Internet. It offers chance to the customers to put all workload and services into cloud and gets all the set of services from cloud. The general objective of cloud computing is to give on-demand computing services which are exceptionally scalable, reliable and open in distributed environments. Reliable QoS (Quality of Service) is one of the real deliverable in a Cloud computing environment. These properties can be portrayed in the Service Level Agreements (SLA) with properties as minimum throughput, maximum latency delivered by the conveyed system [2].

Cloud computing refers to the utilization of cloud or Internet-based PCs for a variety of services. This marvel makes the remote collaboration less demanding. Storage furthermore, uses of data will be basically changed in cloud computing situation [3]. The service models of cloud are classified as Platform-as-a-Service, Software-as-a-Service, and Infrastructure-as-aService. The deployment models are to be specific private, community, hybrid, and public Clouds. The cloud characteristics incorporate pay per use or estimated on-demand service, asset pooling, broad network access and elasticity.

As a huge number of organizations are moving their workload to the cloud nowadays because of this reason the cloud services are to be made accessible on-demand in the ideal time. The shifting of workloads by the associations and even people give rise to the issue of energy utilization and carbon emissions issues from the cloud related infrastructures. The cloud data centers add to this energy consumption in a high amount. The cloud infrastructure comprises of thousands of data centers which satisfy the different demands of the customers online and on time. These data centers involve hundred to thousand feet of area.

Immense measure of power is required for running these servers, cooling fans of network peripherals, processors, console, monitors, light and cooling system. The total power expended utilized by these data centers was around 38 Giga Watt (GW) in 2012 [4] which were sufficient to fulfill the energy requirements of every single resident hardware of UK if it was used properly by them. Contrasted with 2011 the power utilization was around $63 \%$ and more than that. It was around $43 \mathrm{GW}$ in 2013 and more than this in 2014. Since the cloud computing has a capacity of giving on demand services, the requirement for the same is expanding nowadays, numerous ventures moved their services on to cloud, this is the place the data centers comes into the picture.

Mark Hachman [1] in his statistics told that all data centers around the globe consumed electricity in the units of 30 billion of watts in 2012 which is equivalent to productivity from more than 25 nuclear power plants. To chill off these servers, it requires power that can be utilized as a part of 5 million households for 1 year and server farm in 1 year. Here comes the need of energy effective computing or the prerequisite of green computing into the photo of cloud computing. The principle subject of energy effective computing is to profit computing with highlight on reliability, scalable, dynamic and on demand properties. Through cloud and virtualization we can achieve these properties. One of the techniques to diminish the power utilization is to disperse the workload among various servers based on time and space. Other route for decreasing the power utilization is to diminish the CPU use and other sit still resources. The statistics say that even with $10 \%$ of CPU usage, the total power utilization came to up to $50 \%$ at the peak [5].

Cloud Computing contains three service delivery and deployment models as shown in Fig. 1. The delivery model are as follows: 
Public Cloud: Cloud environment which is available to the cloud users for using the infrastructure by registering in the cloud.

Private Cloud: Cloud platform which is for specific organization.

Hybrid Cloud: Private Cloud which uses the resources of the Public Cloud.

Delivery models are as follows:

Software-as-a-Service (SaaS): Cloud provider's give's applications to host and implement in cloud infrastructure.Platform-as-a-Service (PaaS): Cloud provider's delivers platform, tools and business service to develop, deploy andmanaging the applications.

Infrastructure-as-a-Service (IaaS): Cloud provider delivers storage and network resource computation.

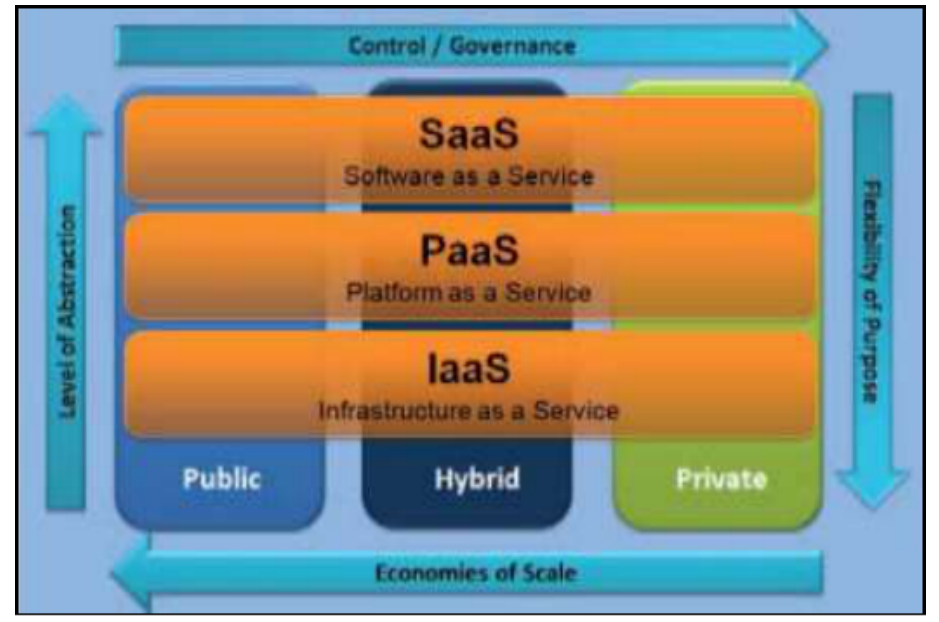

Figure.1 Cloud Computing Model

\section{ENERGY CONSUMPTION ISSUES}

In the previous couple of years, cloud computing has increased much popularity in sharing resources and services through internet along with characteristics, for example, rapid, accessibility, reliability, and so on. The developing demand for these services has expanded the usage of power utilizations in numerous server farms and other cloud related infrastructures.

\subsection{Data centers and other Cloud Infrastructures}

Every data centers focuses incorporate a large number of servers, power transformers and other power expending equipment. For instance, the national security agency which has been building data centers in Utah is required to expend more than 70 megawatts power. Expanding cost of power utilization in data centers has emerged the new requirement for advances and algorithm to limit information enters energy use [6]. As indicated by a review led in the US, just $50 \%$ of the power expended can be added to valuable work done by servers, lay continues cooling, infrastructures, and so on. Power Usage Efficiency (PUE) analyzes how much vitality is utilized by computing and infrastructure hardware. Perfect efficiency would give PUE of 1.0. Most data centers are in the scope of 1.3 to 3.0. Reports have assessed that exclusive $13.4 \%$ of associations screen their utilization data.

As the need of cloud computing is ascending because of its capacity of giving on demand services, numerous organizations are shifting their services on cloud, more data centers are coming in picture. One data centers that involves 50,000 square feet requires 5 megawatt $(\mathrm{mw})$ power that is adequate to power 5,000 households for one year. The cooling requirement of these data centers comprising of thousands of servers is likewise substantial, these servers run 
every one of the 365 days for full 24 hours to give nonstop service although all the servers are not used constantly but rather they can't be turned off because of the cloud service providing phenomena. Mark Hachman [1] in his statistics told that all server farms far and wide expended power in the units of 30 billions of watts in 2012 which is equivalent to the efficiency from in excess of 25 nuclear power plants. To chill off these servers it requires power that can be utilized as a part of 5 million households for 1 year and data center in 1 year.

\subsection{Population and Energy Consumption}

The general energy utilization increments because of the expansion in the population. According to the statistics it is watched that despite the fact that Americans constitutes just 5\% of the world's population however they devour $20 \%$ of its energy. If so then the future age will be left with less measures of resources and furthermore the effect of this will be intense on all the living beings in the earth. When it comes to the cloud computing background, the organizations and people who are shifting their workload to the cloud additionally tend to build nowadays. Because of this shifting, the energy consumed by the data centers to execute the different undertakings given by the clients thusly prepare for high energy utilization. High energy utilization by the data center will deliver a lot of carbon footprints. The carbon emissions will have an awful effect on the environment. So the requirement for energy efficient computing needs to do with the lessening of energy delivered and additionally looking on the issue of population.

\subsection{Modeling Flaws}

Modeling flaws may exist because of different reasons, for example, the exaggerated adoption costs, incorrect discount rates, and possibility of not being real and so forth. The overstated adoption costs happen because of second rate venture execution as per presumptions, and poor policy plan. At the point when the customers are provided with incorrect rates as indicated by the present market rates and the supplier cases to give discount the incorrect discount rate issue arises. The clients misconstrue the rates and get the service and later on suffer with the harmful effects. The possibility of not being real can likewise be contrasted and the past situation. An additional point is that the client may get fooled saying that the specific service offered may act especially later on however carries on in an unexpected way. In this way modeling flaws can be considered as one reason for energy utilization as the client gets fooled about the model or the service the supplier claim to provide.

\section{LITERATURE SURVEY}

In this section we presents existing work done in the field of energy efficient cloud computing model.

Shahin et al. [7] proposes a scheduler which implements the cut-and solve-based algorithm and the call back method are proposed to reduce the complexity as well as computation time. Proposed work enhances the state of the art in workload estimation and dynamic power management of cloud DCs, and the results will be helpful to cloud service providers in achieving energy optimization.

Liu et al. [8], implemented an optimized scheduling methodology to reduce energy optimization while fulfilling task reaction time limitations amid scheduling. This methodology is an insatiable approach that chooses the base number of the most efficient server for scheduling. The undertakings are heterogeneous in nature with the end goal that they constitute diverse energy utilization levels and have different task reaction times. The ideal task depends on least energy optimization and least fulfillment time of a job on a specific machine. 
Kliazovich et al. [9], proposed DENS or server farm energy-efficient system network-aware scheduling. In this framework, the scheduling of tasks is performed by consolidating system awareness and energy productivity. DENS fulfills QoS requirements and improves work processes. This framework lessens the quantity of computing servers and keeps away from hotspots. System awareness is gotten by utilizing feedback channels from the principle network switches. This technique has less computational and memory overhead.

Kliazovic et al. [10], proposed e-STAB or Energy-Efficient Scheduling for Cloud Computing Applications with traffic load balancing. The analysts basically centered on energyefficient job scheduling that considers traffic load adjusting in cloud data centers. They likewise took a gander at the movement necessities of cloud applications. e-STAB limits congestion and communication delays in the system.

Liu et al. [11], presented the Adaptive Energy-efficient Scheduling (AES) system, which joins the Dynamic Voltage Scaling (DVS) procedure with the versatile tasks duplication technique. In the principal stage, a versatile edge based tasks duplication procedure is proposed, which can provide an optimal threshold. In the second stage, the groups are planned on DVSempowered processors to reduce processor energy at whatever point tasks have slack time because of dependencies. This algorithm can adequately optimize energy while keeping up great execution.

Mehdi et al. [12], proposed a two-stage minimum completion algorithm (2PMC) that chooses machines for task scheduling in light of the normal minimum completion time of each single accessible machine. This algorithm thinks about the load of the machine before scheduling the tasks. The completion time of a task on the machine can be defined as the sum of the execution time of the task on that machine and the preparation time of that particular machine.

\section{PROBLEM IDENTIFICATION}

The issue close by in this work is to offer a basic, sensible and open source test system to help investigate on energy optimization in Clouds running work processes. The specific parts of this issue can be sketched out as follows:

The test system must give DVFS capacities controlled by the standard sorts of governors to Cloud server farms. DVFS [13] is a key procedure for energy optimization in Cloud Computing, as showed up by the present ascent of various energy optimization systems that are being made considering DVFS-empowered composed server farms (e.g., jobs scheduling). Generally, DVFS is a method that, in light of the type of senator, changes recurrence levels of handling components as indicated by the measure of workload to be executed [14].

Once the execution of the workload has finished, it must offer an estimation of the by and large and normal power and devoured up energy and furthermore of make span. If a test framework can't give an estimation of these parameters, it is illogical to illuminate the Cloud administrator or customer about the execution of the outline framework and thusly, the procedures for energy saving can't be broke down and advanced. To deal with this issue, it is vital to get a practical model and facilitate it in the test system. Besides, it is important that the power indicate does not simply consider the figuring cost of the preparing components moreover, the reconfiguration usage in one of a kind DVFS governors and the system costs, as later and productive works in Cloud Computing purpose. 


\subsection{Proposed MEthodology}

The methodologies for energy saving in server farms can be requested into host (or intra-host) and networks (or between host) levels. While the network level energy careful strategies are essentially established on the coordination and participation of machines through booking systems to decrease control use, the host level ones are committed to increase inside-machine proficiency.

This work concentrates on the audit and usage of the host energy careful DVFS system for genuine Cloud simulation systems. DVFS offers a capable and persistent way to deal with decrease the scattered power in a processor by changing its clock speed and the provided voltage during both the periods of inertness and escalated registering in applications execution.

Using this system generous declines in power use with a greatly slight loss of adequacy in its execution are expert. Distinctive superior figuring stages in light of server farms, for example, group registering and supercomputing apply DVFS advances to decrease control usage and to get a high unwavering quality and accessibility in their establishments.

\subsection{Workflow Execution Task}

Workflow Simulator implements CloudSim to executes the workloads where given jobs have complex dependencies. We are experiment Sipht (30 jobs), exhibit as DAG. This paper followed, these work processes and executes on the virtual machine by means of CloudSim. The dependencies can be seen in fig. 4.1 for Sipht.

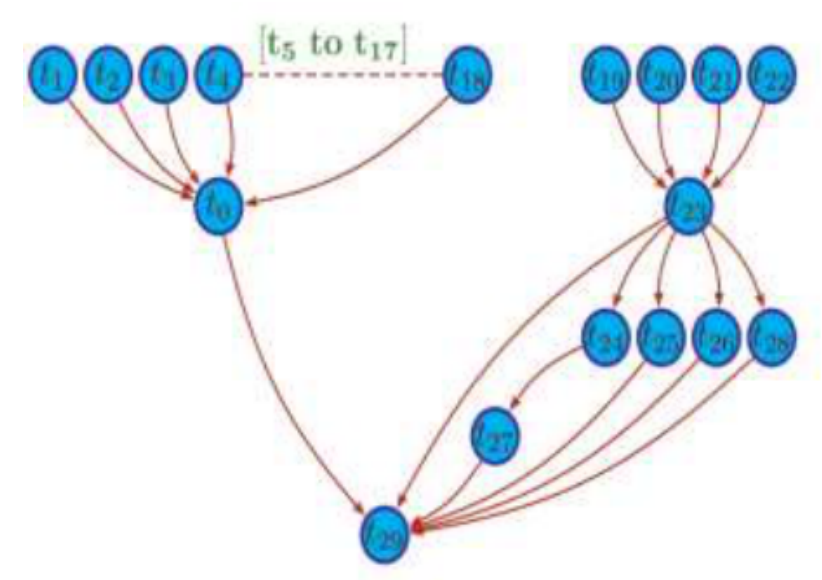

Figure. 2. Sipht Workflow

Firstly it takes, the tasks are send to the merger. Generally, this stage groups the task as small jobs. After that these small jobs are sent to the engine module, they are executed in the order indicated by the work process. Engine modules makes each job to take after the predefined work process so the dependencies are resolved accurately without any collision. Finally, the scheduler select one of the VM from the cloud farm which is more reasonable for handling these work process. Fig. 2 speaks to graphically the exchange of messages between various modules in each stage. 


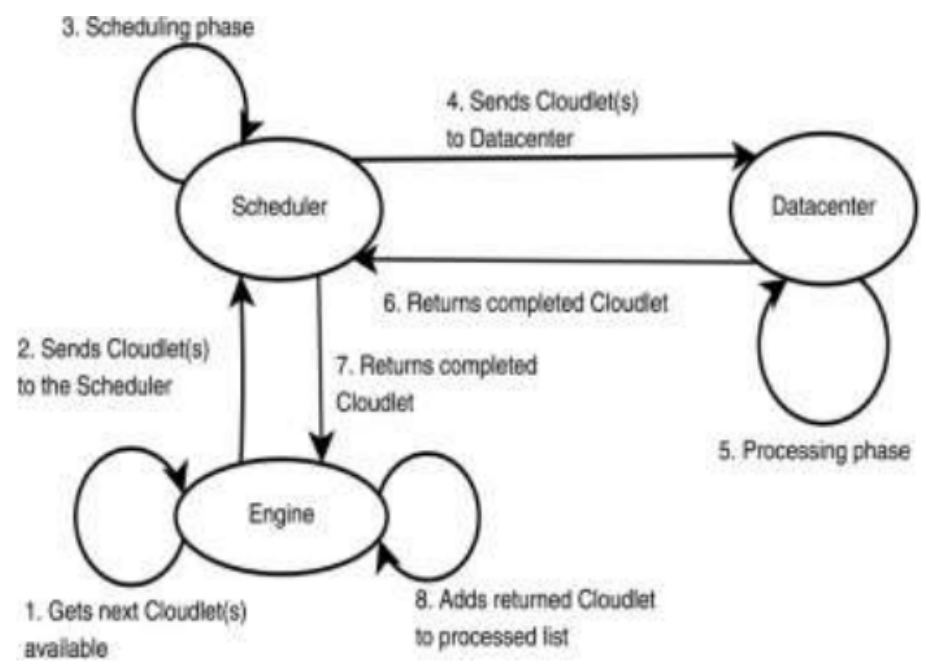

Figure. 3. Shows the proces of main stage

\subsection{Mode of Execution}

The Governor can process the jobs in two different ways:

1. OnDemand

2. Conservative

OnDemand Mode: When CPU load is uncertain at execution organize, OnDemand mode is applied. It favors host by expanding CPU frequency to its maximum capacity when CPU load exceed the predefined threshold. When CPU load drops down the threshold, the CPU again scale down step by step.

Conservative Mode: This mode increment the frequency step by step with some predefined record. It generally follow some record to increase the frequency and scales down step by step.

\section{RESULT AND DISCUSSION}

To assess our metrics we have utilized Cloud Simulator. Cloud Simulator tools are utilized to simulate the jobs by giving execution in cloud computing condition. We have taken Sipht work process dataset which are accessible online.

Table I demonstrates the specification of our experiment setup.

TABEL I: Experiment Setup Configuration

\begin{tabular}{|c|c|c|}
\hline S.No. & Attributes & Value \\
\hline 1 & Simulator & CloudSim \\
\hline 2 & Language & JAVA \\
\hline 3 & $\begin{array}{c}\text { Algorithm } \\
\text { Used }\end{array}$ & DVFS with Migration Manager \\
\hline 4 & Dataset & Sphit Workflow DAX \\
\hline 5 & $\begin{array}{c}\text { Operating } \\
\text { Mode }\end{array}$ & Conservative and OnDemand \\
\hline
\end{tabular}


We have analysed our algorithm on onDemand and Conservative mode of operation. The outcome demonstrates that in conservative mode in Sipht dataset takes 2823 energy while in OnDemand takes 2717 energy. Conservative is 1.039 times costlier than OnDmand mode in Sipht.

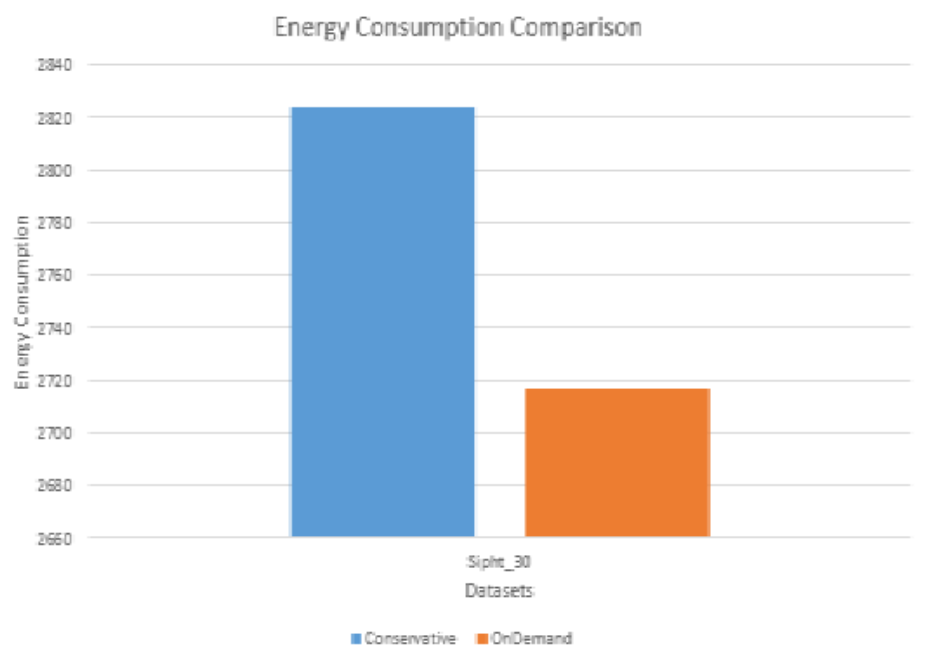

Figure. 3. Shows the comparison between Conservative and OnDemand Mode of Execution

\section{CONCLUSION}

The purpose behind this paper is to give an effective method to schedule the resources into various VM's. The working of migration manager is described briefly that how information are exchanged starting with one VM's then onto the next VM's without affecting the overall performance. We have involved DAX and DVFS algorithm into CloudSim. We have exhibited the onDemand and Conservative mode and suggested that OnDemand mode is more efficient than conservative mode while migrating resources and additionally in terms of optimizing energy.

\section{REFERENCES}

[1] “Datacenters". (2015, May 10) [Online] Available:http://slashdot.org/topic/datacenter/newyork-timestakes-aim-at-datacenters.

[2] "Cloud Computing". (2015, May 10) [Online] Available: https://en.wikipedia.org/wiki/Cloud_computing.

[3] B. Damien, M. Michael, D.-C. Georges, P. Jean-Marc, and B. Ivona, "Energy-efficient and SLA-aware management of IaaS clouds," in 3rd International Conference on Future Energy Systems: Where Energy, Computing and Communication Meet, Madrid, Spain, 2012.

[4] "Taxes and incentives for renewable energy", KPMG international, June 2012.

[5] C. Yang, K. Wang, H. Cheng, C. Kuo, W.C. Chu, "Green Power Management with Dynamic Resource Allocation for Cloud Virtual Machines", IEEE International Conference on High Performance Computing and Communications, 2011.

[6] M. Ghamkhari and H. Mohsenian-Rad, "Energy and Performance Management of Green Data Centres: A Profit Maximization Approach", IEEE Transactions on Smart Grid, Vol. 4, No. 2, pp. 1017-1025, 2013.

[7] S. Vakilinia, B. Heidarpour and M. Cheriet, "Energy Efficient Resource Allocation in Cloud Computing Environments," in IEEE Access, vol. 4, pp. 8544-8557, 2016. 
[8] Liu, N., Dong, Z., \& Rojas-Cessa, R. (2013, July). Task scheduling and server provisioning for energy-efficient cloud-computing data centers. In Distributed Computing Systems Workshops (ICDCSW), 2013 IEEE 33rd International Conference on (pp. 226-231). IEEE.

[9] Kliazovich, D., Bouvry, P., \& Khan, S. U. (2013). DENS: data center energy-efficient network-aware scheduling. Cluster computing, 16(1), 65-75.

[10] Kliazovich, D., Arzo, S. T., Granelli, F., Bouvry, P., \& Khan, S. U. (2013, August). eSTAB: energy-efficient scheduling for cloud computing applications with traffic load balancing. In Green Computing and Communications (GreenCom), 2013 IEEE and Internet of Things (iThings/CPSCom), IEEE International Conference on and IEEE Cyber, Physical and Social Computing(pp. 7-13). IEEE.

[11] Liu, W., Du, W., Chen, J., Wang, W., \& Zeng, G. (2014). Adaptive energy-efficient scheduling algorithm for parallel tasks on homogeneous clusters.Journal of Network and Computer Applications, 41, 101-113.

[12] Mehdi, N. A., Ali, H., Amer, A., \& Abdul-Mehdi, Z. T. (2012). TwoPhase Provisioning for HPC Tasks in Virtualized Datacenters. In Proc. International Conference on Emerging Trends in Computer and Electronics Engineering (ICETCEE), Dubai.

[13] D. L. swets and J. Weng. "Using discriminant eigen features for image retrieval". IEEE Trans. PAMI, 18(8):831-836, 1996.

[14] Z. Hong. "Algebraic feature extraction of image for recognition". Pattern Recognition, 24(2):211-219, 1991 